\title{
Underground Imaging by Sub-Terahertz Radiation
}

\author{
Yuan Zheng ${ }^{1}{ }^{1}$, Calvin Domier ${ }^{1}$, Michelle Gonzalez ${ }^{1}$, Neville C. Luhmann, Jr. ${ }^{1}$ and Diana Gamzina ${ }^{2, *}$ \\ 1 Department of Electrical and Computer Engineering, University of California, Davis, CA 95616, USA; \\ zyzheng@ucdavis.edu (Y.Z.); cwdomier@ucdavis.edu (C.D.); mgon@ucdavis.edu (M.G.); \\ ncluhmann@ucdavis.edu (N.C.L.J.) \\ 2 The Technology Innovation Directorate, SLAC National Acceleration Laboratory, Menlo Park, CA 94025, USA \\ * Correspondence: dgamzina@slac.stanford.edu
}

check for

updates

Citation: Zheng, Y.; Domier, C.;

Gonzalez, M.; Luhmann, N.C., Jr.;

Gamzina, D. Underground Imaging

by Sub-Terahertz Radiation.

Electronics 2021, 10, 2694.

https://doi.org/10.3390/

electronics10212694

Academic Editor: Joaquin Portilla

Received: 22 September 2021

Accepted: 2 November 2021

Published: 4 November 2021

Publisher's Note: MDPI stays neutral with regard to jurisdictional claims in published maps and institutional affiliations.

Copyright: (c) 2021 by the authors. Licensee MDPI, Basel, Switzerland. This article is an open access article distributed under the terms and conditions of the Creative Commons Attribution (CC BY) license (https:/ / creativecommons.org/licenses/by/ $4.0 /)$.

\begin{abstract}
Sub-terahertz ground-penetrating radar systems offer an alternative to radio wave-based systems in the airborne imaging of buried objects. Laboratory prototype systems operating in $\mathrm{W}$-band (75-110 GHz) and F-band (90-140 GHz) are presented, detecting the distance between target and source and imaging metal objects buried in mixed soil. The experimental results show that imaging in the 100-150 GHz frequency range is feasible for underground applications but significantly restricted by the attenuation characteristics of the medium covering the targets. A higher power source and more sensitive receiving components are essential to increase the penetration capability and expand the application settings of this approach.
\end{abstract}

Keywords: GPR; landmine detection; millimeter wave imaging

\section{Introduction}

The SLAC National Accelerator Laboratory (SLAC) in collaboration with the UC Davis Millimeter Wave Research Center developed a technique for underground millimeter wave imaging. When successfully developed and applied in actual conditions, this technique would enable reliable and fast imaging of underground $(30 \mathrm{~cm})$ as well as above-ground objects, in addition to being able to discern variation in soil density due to the system's high resolution. Such a multifunctional imaging technique can be adapted to a variety of operating environments and could also target a wide spectrum of applications including (but not limited to) landmine imaging [1]. The demonstration of the high-resolution capability of the millimeter wave radiation would lay the foundation for a potential future concept demonstrator, which would utilize technological advances in compact highpower transmitters [2-4] and fully integrated receiver systems on a chip ("system-on-chip": SoC) [5]. After successful demonstration, the technique could potentially be employed in an ultra-compact, fully integrated, remotely controlled and UAS (Unmanned Aircraft Systems) - compatible landmine and underground imaging system enabling fast, highreliability landmine image generation. Algorithms could then be employed to train the system to compare and overlay images in real time. The future UAS compatible system would employ transceivers for the generation and collection of the signals, while utilizing an ultra-lightweight high-power $(\sim 500 \mathrm{~W})$ broad-bandwidth $(\sim 30 \mathrm{GHz})$ travelling wave tube amplifier for signal amplification at high frequencies. Such a combination would offer sufficient resolution (few millimeters) and power for target imaging down to about $30 \mathrm{~cm}$ of dry soil depths and with about $2 \mathrm{~m}$ of above-ground stand-off. The future system would employ pulse-shaping techniques to transmit and collect both time-domain and frequency-domain data, significantly enhancing the resolution and sensitivity of the system. The imaging overlay technology would then generate high-resolution, three-dimensional images of the soil.

Ground-penetrating radar is a technique used to locate sub-surface objects through the illumination of the ground by either pulsed or frequency-swept electromagnetic waves, 
using variations in the signals reflected or scattered from a buried object to determine its position within the ground [6]. Operation at microwave frequencies is shown to have good depth penetration, albeit with limited resolution that scales with the wavelength of the probing radar beam [7]. The use of sub-terahertz radiation, with its significantly shorter wavelengths, opens up the possibility for high-resolution sub-surface imaging, although with a shallower penetration depth.

For improved resolution, a synthetic aperture radar (SAR) approach or, more accurately, an underground SAR approach, is often employed [7]. Whereas a real aperture radar (RAR) has an azimuth resolution determined by the scanning beam's footprint on the ground, the resolution of an SAR system is improved by analyzing the return signal from multiple reflections that are spatially separated from each other to effectively synthesize a larger antenna with a much narrower beamwidth. The sampled points must be spaced closer than $\lambda / 2$, where $\lambda$ is the wavelength of the probing beam, and the antenna position must be known with an accuracy better than $\lambda / 4$ azimuthally and $\lambda / 8$ in range [8,9]. The $<\lambda / 4$ positional accuracy requirement of SAR means that, as the radar frequency increases (and the wavelength decreases), the position of each measurement location must be known with ever increasing precision. While this can be easily achieved in the laboratory frame, it is harder to accomplish in the outdoors with ground-based measurement systems and even harder with airborne systems, as illustrated in Figure 1. Recent advances in the field of airborne landmine detection systems have employed Real-Time Kinematic positioning systems with a positional accuracy as good as $10 \mathrm{~mm}[9,10]$, sufficient for underground imaging systems operating at a maximum frequency of about $6 \mathrm{GHz}$.
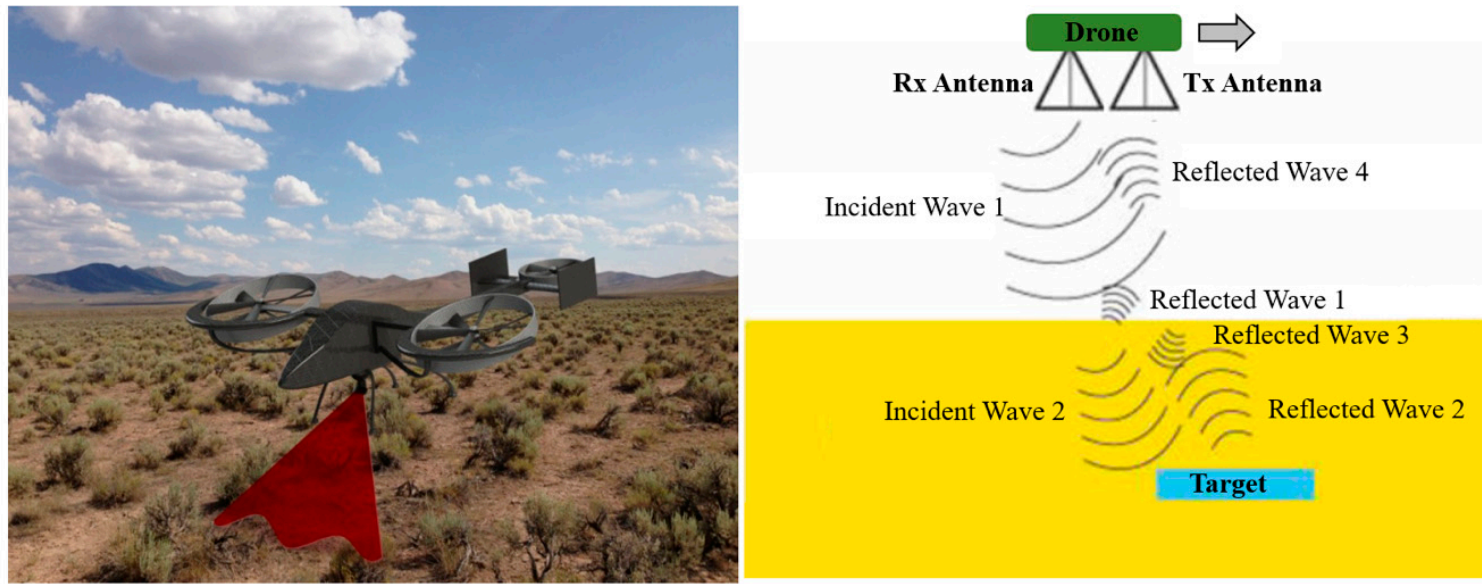

Figure 1. Airborne high-power sub-Terahertz-radiation underground imaging.

In this paper, we describe an alternate approach to high-resolution underground imaging using sub-terahertz radiation. These initial measurements employed a simple RAR configuration using a more tightly focused beam to maximize the received power to help overcome the enhanced soil attenuation levels observed at higher frequencies. Extending this technique to a SAR approach will be possible at a later date and is not included herein.

\section{Sub-Terahertz Swept-FM Imaging Experimental Validation}

The prototype W/F-band (75-110/90-140 GHz) swept-FM systems are shown schematically in Figure 2. An HP 83752A synthesized sweeper drove an active X6 multiplier chain (AMC) to generate the probe beam that was swept from $80 \mathrm{GHz}$ to $100 \mathrm{GHz}$, with >+12 dBm W-band output power across the sweep. A V-band X4 (50-75 GHz) AMC and an F-band X2 (90-140 GHz) passive-frequency multiplier (PFM) combination was used to replace the $\mathrm{W}$-band AMC and comprise the system operating at $\mathrm{F}$ band. A portion of the outgoing beam was coupled out to the receiver, which employed a single-ended Schottky diode detector which mixed this signal with that of the time-delayed echo pulse collected by a horn 
antenna. The detector output eas then amplified and filtered using a $10 \mathrm{kHz}$, low-pass and a variable-frequency high-pass filter. The resultant signal was viewed on an oscilloscope and simultaneously sampled for transfer to LabVIEW for analysis. Also displayed and digitized is the Sweep Out signal from the sweeper (voltage ramped from $0.0 \mathrm{~V}$ to $2.0 \mathrm{~V}$ during the sweep), which allowed LabVIEW to determine when the sweep was taking place. In swept-FM reflectometry, the probe source is frequency-modulated (FM) so that its frequency is swept or monotonically increased over the length of the sweep. The reflected wave packet or echo is fed to a mixer that is pumped by a portion of the outgoing probe wave. In the case of a linear-frequency sweep, the intermediate frequency $f_{I F}$ of the mixer output signal is then linearly proportional to the time delay between the probe and echo signals, as shown in the dash frame of Figure 2. The range $R$ or distance to the target can then be calculated by using:

$$
f_{I F}=(2 R / c) \times(\Delta f / \Delta t)
$$

where $c$ is the speed of light, and $d f / d t$ is the rate at which the frequency changes in time.

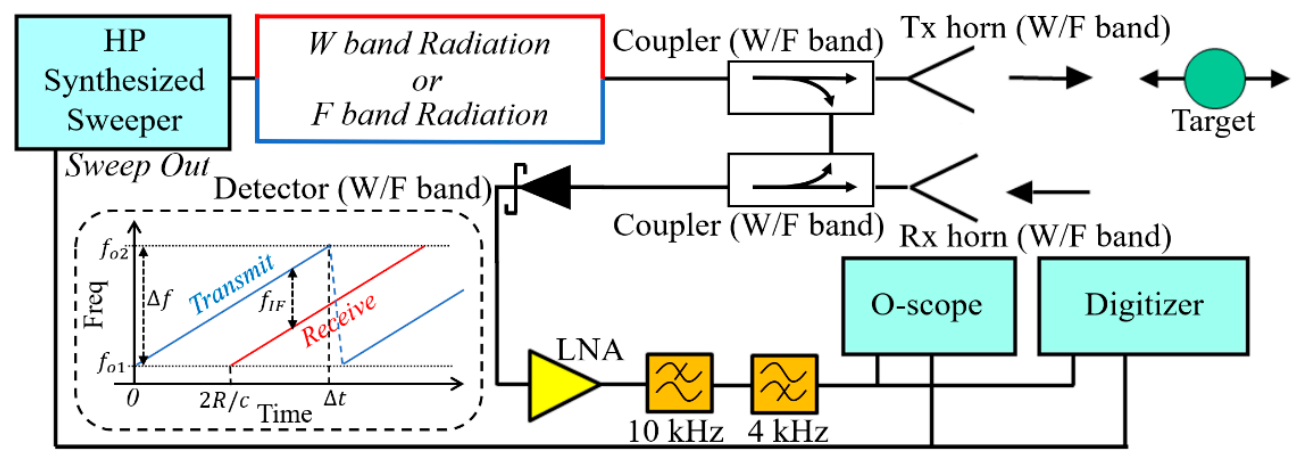

Figure 2. Schematic representation of the prototype W/F-band swept-FM radar system.

\subsection{1-D Range Measurements}

In the swept-FM system, a receiver mixes the detected echoes with a portion of the outgoing beam, with the result that echoes from different depths end up with different IF frequencies after mixing. This offers the potential to discriminate between the various echoes using analog and/or digital filtering. The W-band swept-FM system prototype, using a $+12 \mathrm{dBm}$ or $15 \mathrm{~mW}$ frequency multiplier source, is shown in Figure 3 . The probe beam was generated by a $20 \mathrm{dBi}$ gain horn antenna equipped with a collimating lens to reduce the spot size of the probe beam near the 'target', with the reflected radiation collected by a slightly larger $25 \mathrm{dBi}$ gain horn. It should be noted that all measurements took place in the near field of the horn antennas where the far field beam patterns were not sufficient to properly characterize the azimuthal characteristics of the probe beam near the target. For these initial measurements, the target was a $25 \mathrm{~mm}$-diameter aluminum rod attached to the top of a slide that permitted $>230 \mathrm{~mm}$ of travel. Also shown in the left photo of Figure 3 is a thin wooden board which represents the surface of the sand/soil; the wooden board rested $25 \mathrm{~mm}$ in front of the target at its closest to the radar system. This wooden board provided a fixed reflection and attenuated the beam by roughly $2 \mathrm{~dB}$ in both directions. In this setup, the probe frequency was swept from $82.2 \mathrm{GHz}$ to $99.6 \mathrm{GHz}$ in $9.95 \mathrm{~ms}$, with an average sweep rate of $1.75 \mathrm{GHz} / \mathrm{ms}$. We assume that the probe and the echo signals had an effective path length difference of additional $16 \mathrm{~cm}$ with respect to the signal that traveled between the two directional couplers (the true path length difference was somewhat shorter than this due to waves traveling slower in waveguide than in free space). Using the radar range equation described earlier, the expected difference frequency achieved in this setup should follow the relationship $f_{I F}=117 \times(R+8) \mathrm{Hz}$. 


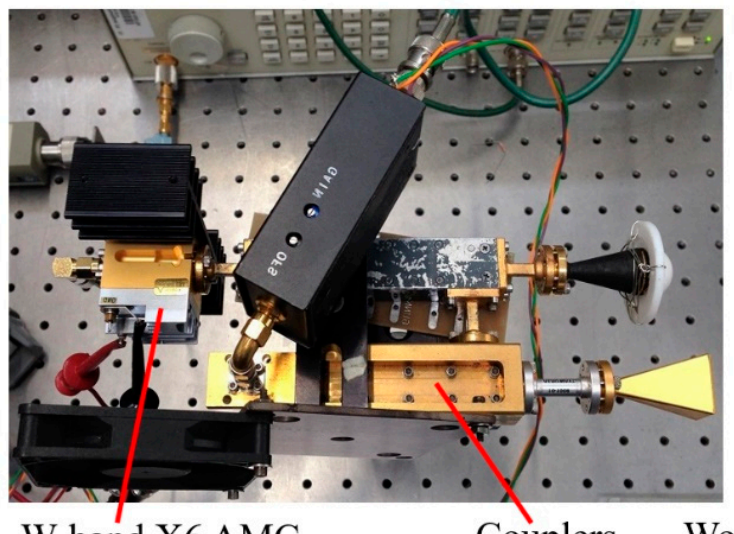

W-band X6 AMC

Couplers

Wood board

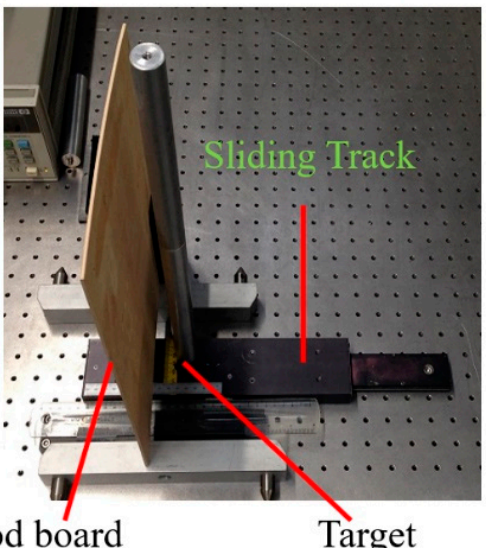

Target

Figure 3. Photographs of the prototype W-band swept-FM radar system and the translatable target region.

The filtered signals were digitized and recorded, after which a 4096-point fast Fourier transform (FFT) was applied. A typical set of signals is plotted in Figure 4a for a single sweep, with the aluminum rod placed $32 \mathrm{~cm}$ from the transmit lens and the wooden board removed. Here, the peak frequency component in the digitized signal was at $4.69 \mathrm{kHz}$ as shown in Figure $4 \mathrm{~b}$, with some higher frequency components detected. The source of these high-frequency components is not clear but is theorized to arise mainly from sidelobe reflections off the translatable target, whose distance (and hence frequency) increased as the target was translated farther from the transmit/receive horns. A similar set of data are presented in Figure 4c, this time at $42 \mathrm{~cm}$, in which the peak frequency component was observed at $5.86 \mathrm{kHz}$, as shown in Figure $4 \mathrm{~d}$. These values compare well with the predicted peak frequencies of $117 \times(32+8)=4.68 \mathrm{kHz}$ and $117 \times(42+8)=5.85 \mathrm{kHz}$.

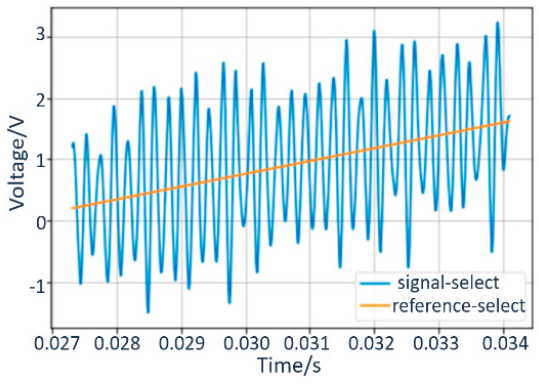

(a)

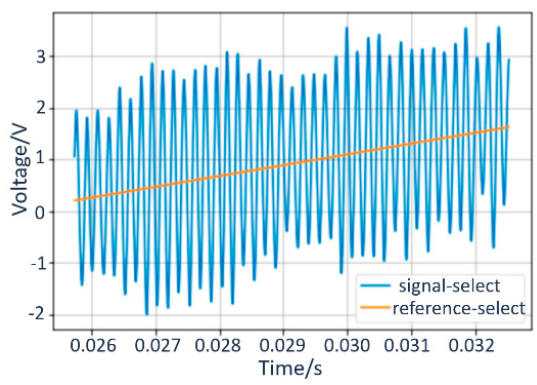

(c)

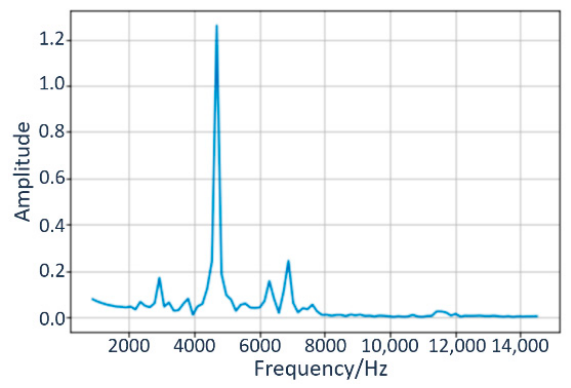

(b)

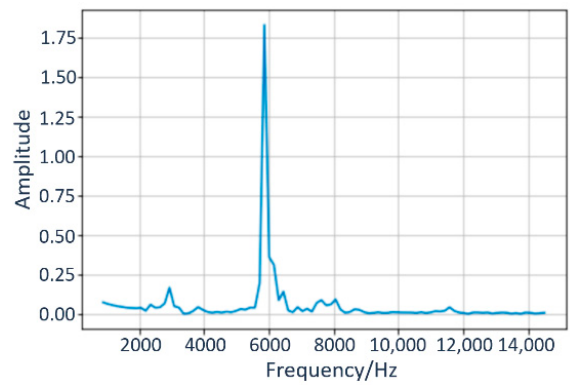

(d)

Figure 4. (a) Output and reference signals observed at a lens-target distance of $32 \mathrm{~cm}$ and (b) FFT of the output signal. A peak signal component of $4.7 \mathrm{kHz}$ is observed. (c) Output and reference signals observed at a lens-target distance of $42 \mathrm{~cm}$ and (d) FFT of the output signal. A peak signal component of $5.9 \mathrm{kHz}$ is observed. 
This experiment was repeated in F-band using a lower power $1.7 \mathrm{~mW}$ frequency multiplier setup and $23 \mathrm{dBi}$ gain horn antennas, as shown in Figure 5. The probe and echo signals had an effective path length difference of additional $10 \mathrm{~cm}$ with respect to the signal that traveled between the two directional couplers in the F band system; the expected difference frequency achieved in this setup should follow the relationship $f_{I F}=200 \times(R+5) \mathrm{Hz}$. Here, the peak frequency component in the digitized signal was at $4.0 \mathrm{kHz}, 5.0 \mathrm{kHz}$, and $6.0 \mathrm{kHz}$ when the aluminum rod was located $15 \mathrm{~cm}, 20 \mathrm{~cm}$, and $25 \mathrm{~cm}$ away from the antenna horn, as shown in Figure 6. The prototype swept-FM system looks promising, with rapid and accurate detection of the aluminum rod even when placed behind a reflective wooden board mocking up a sand/soil surface. The frequency of the detected echo was observed to increase linearly with the distance of the target from the scanner, and it was possible to easily resolve objects that were axially separated by at least $10 \mathrm{~mm}$.

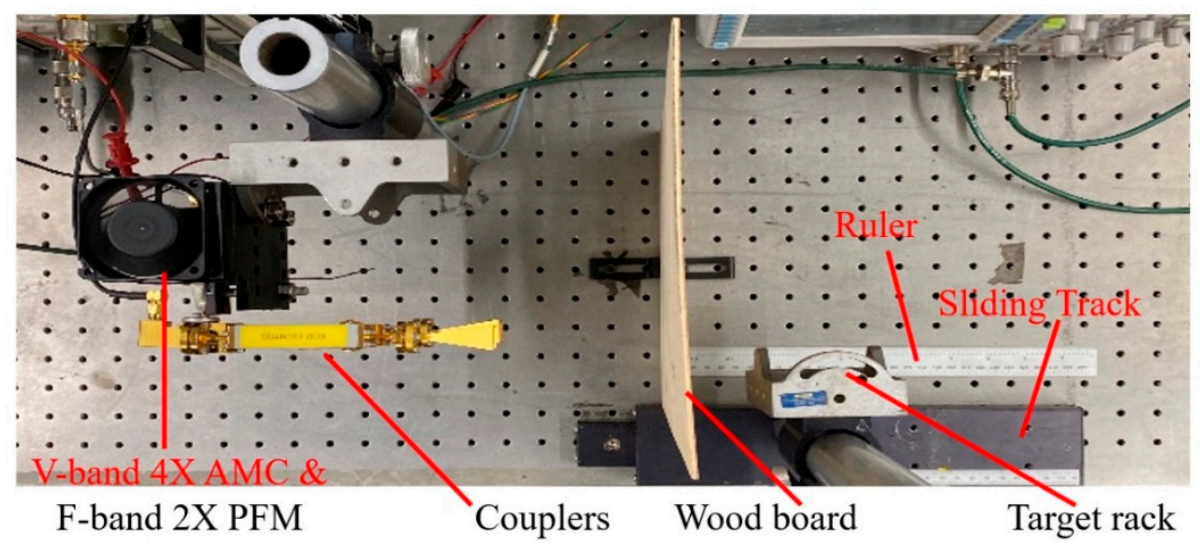

Figure 5. Photographs of the prototype F-band swept-FM radar system and the translatable target region.

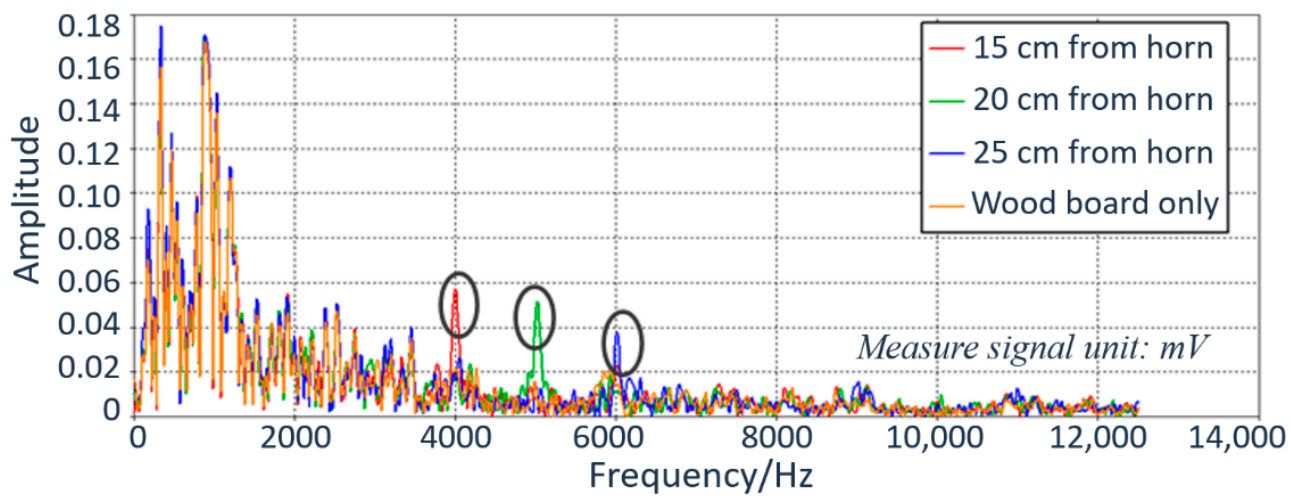

Figure 6. FFT of the output signal of the F-band swept-FM system. Peak signal components of 4.0, 5.0 , and $6.0 \mathrm{kHz}$ are observed when the target distance is 15,20 , and $25 \mathrm{~cm}$ from the antenna horn.

\subsection{Above-Ground 2D Imaging Scans}

In the hope that this swept-FM system could further help to detect some features of the target object benefiting the identification of the target, we mounted the sub-Terahertz radiation source on a $2 \mathrm{D}$ scan platform, as shown in Figure 7, so to imitate a flying drone scanning the ground. Two translation stages were incorporated onto the table: one was introduced along the $x$-axis to hold the second translation stage carrying the imaging system and moving on the $y$-axis, in parallel with the translation stage working along the $x$-axis. The arrangement of the translation stages allowed the imaging system to provide a $30-\mathrm{cm}-\mathrm{by}-30-\mathrm{cm}$ scanning area. Each stage had a resolution of 0.003 inches $(0.076 \mathrm{~mm})$ and was capable of speeds more than $1 \mathrm{inch} / \mathrm{sec}$. LabVIEW was employed to control the stage 
motors and acquire/process the data. The scanning code permitted real-time FFT analysis of the output signal at each location, helping to tune the imaging system.

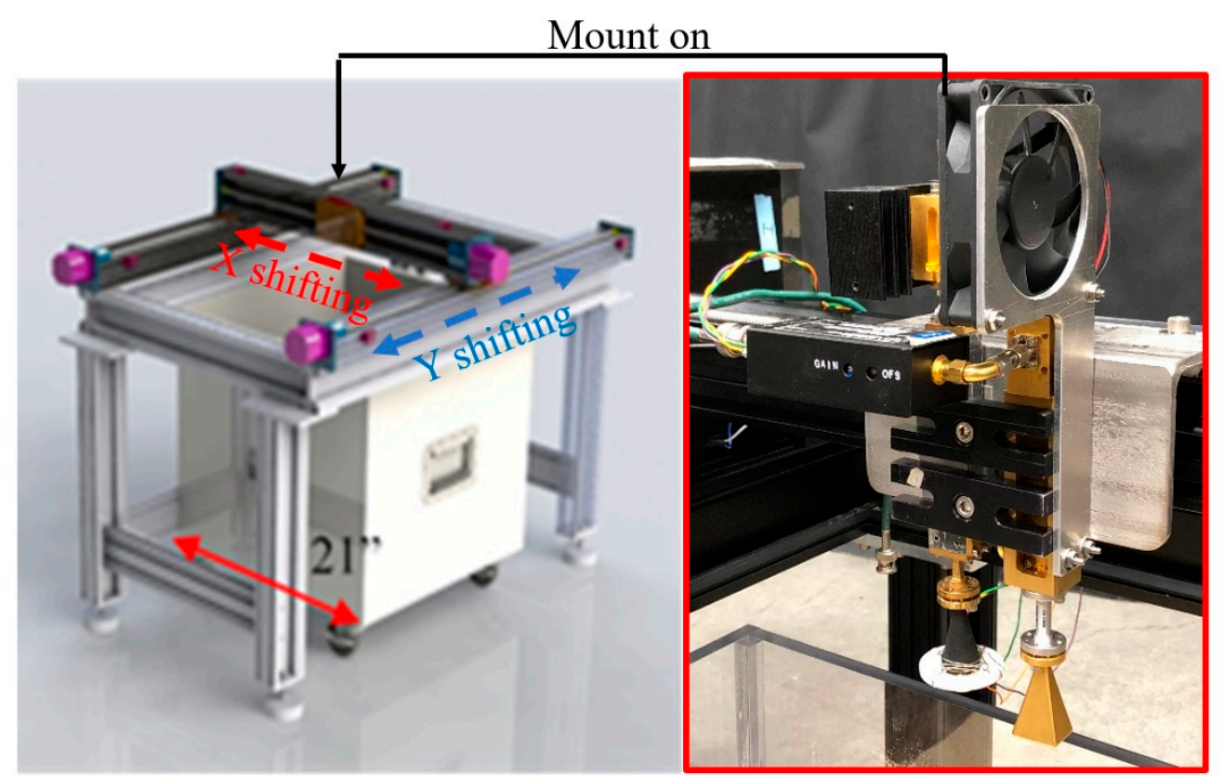

Figure 7. Swept-FM 2D-scan imaging system.

Both the W-band and the F-band swept-FM prototype 2D-scan imaging systems were setup and used to scan the $66 \mathrm{~mm} \times 66 \mathrm{~mm} \times 6.35 \mathrm{~mm}$ square metal plate, as shown in Figure 8. There were two holes and two slots on the plate to verify the resolution of this swept-FM imaging system. In this prototype proof-of-principle verification test, the carbon-loaded absorbing material (Emerson \& Cuming Eccosorb ${ }^{\circledR}$ HR-25) was attached to the target object platform and the surrounding rack to reduce echo wave interference. To balance the imaging speed and resolution, in the following reported experiments, the $2 \mathrm{~d}$-scanning region was $12 \mathrm{~cm} \times 12 \mathrm{~cm}$, and a $2 \mathrm{~mm} / \mathrm{step}$ scanning resolution and a $500 \mathrm{~ms} /$ step scanning speed were adopted.
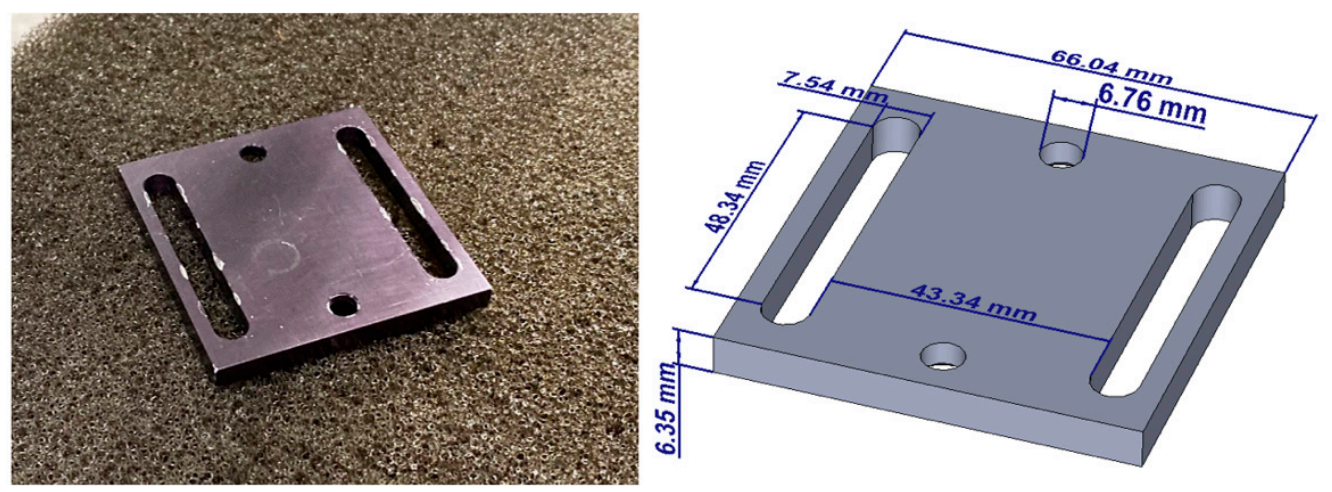

Figure 8. Swept-FM imagine target plate.

The testing plate was placed $\sim 40 \mathrm{~mm}$ from the $\mathrm{W} / \mathrm{F}-$ band radiation source. According to the previous position test, it was predicted to find the target signal around $5.7 \mathrm{kHz}$ and $7.0 \mathrm{kHz}$ on W-band and F-band swept-FM imaging system, separately. The testing results shown in Figures 9 and 10 matched well with the prediction and verified the position accuracy of the swept-FM system. 


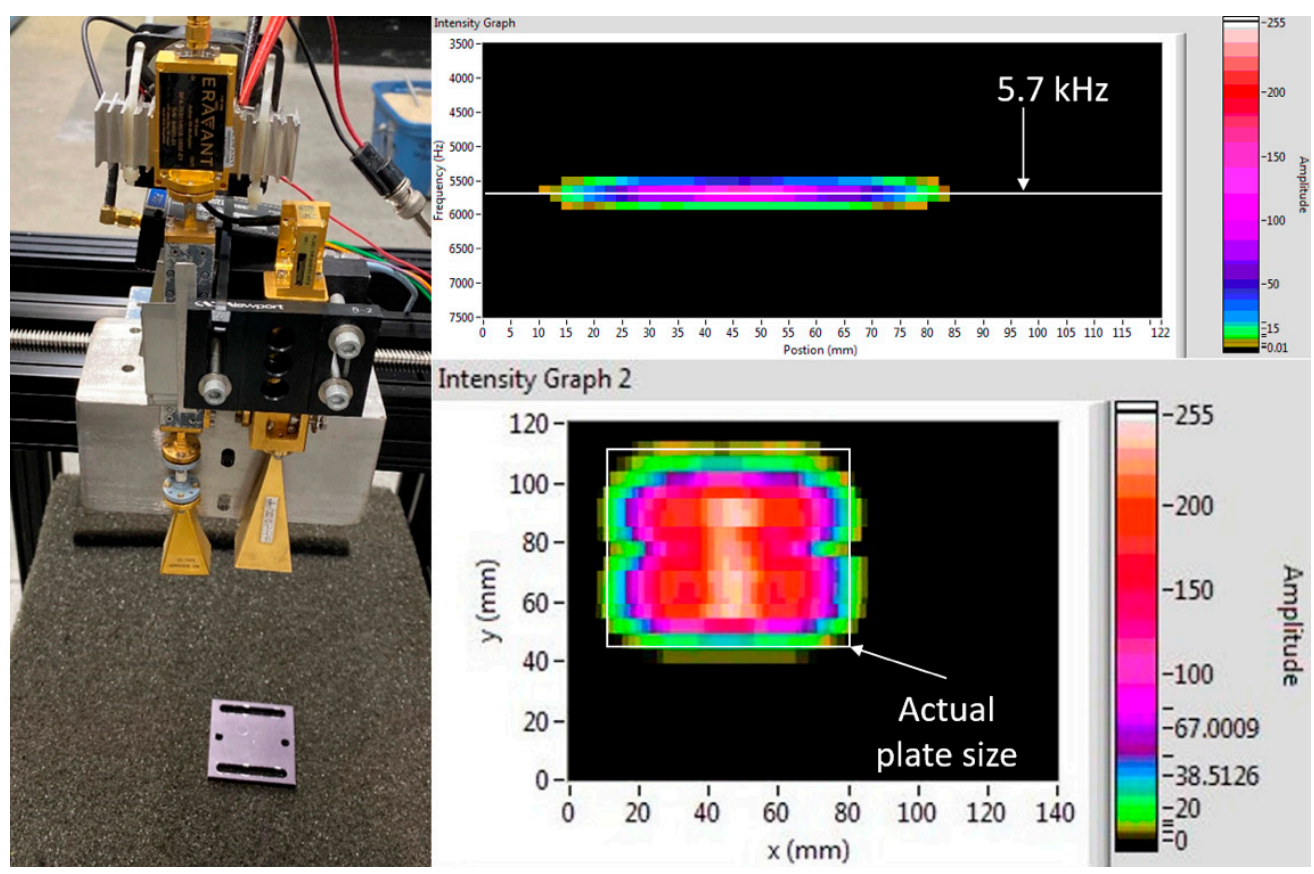

Figure 9. W-band Swept-FM 2D-scan imaging system and related experiment results.

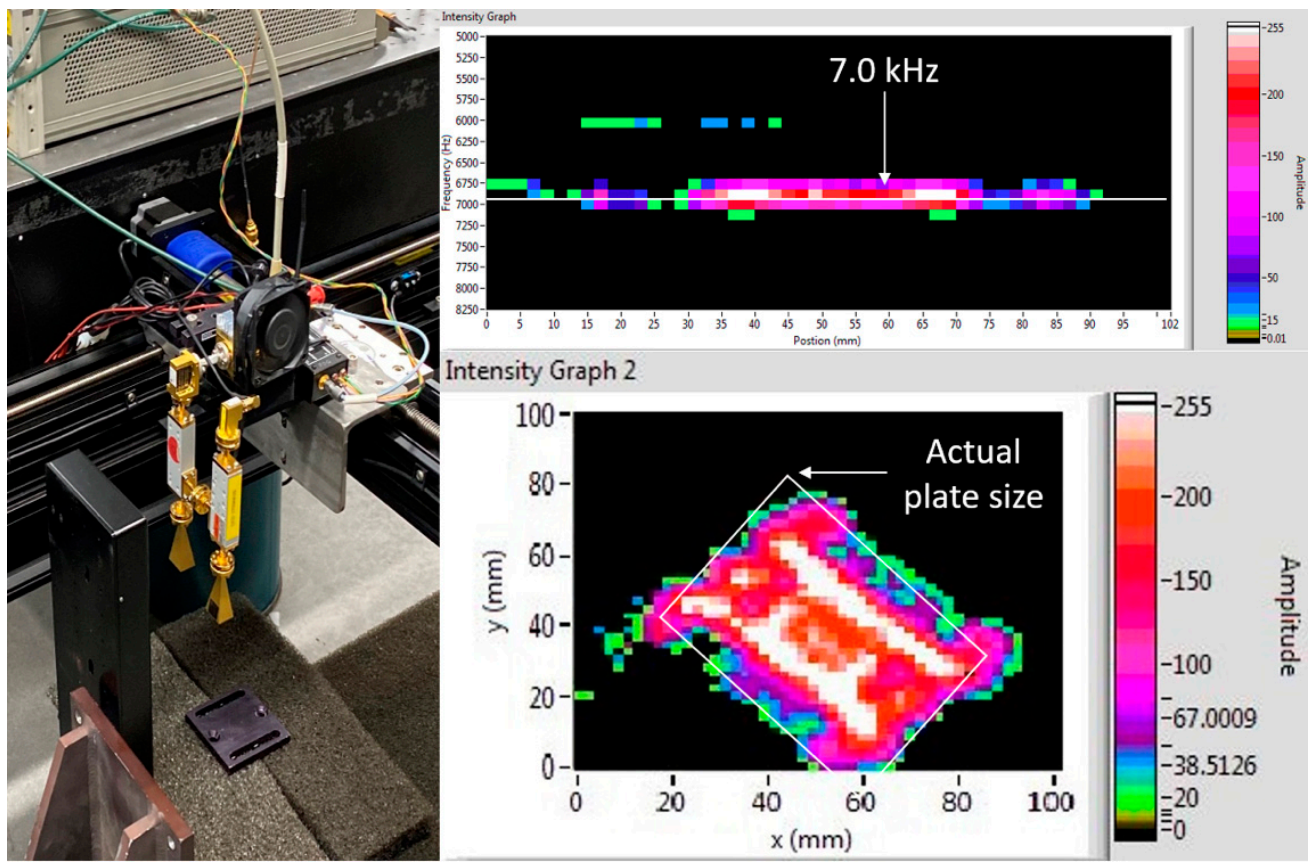

Figure 10. F-band Swept-FM 2D-scan imaging system and related experiment results.

While it was clear from the received spectra that a target was present, both the W-band and the F-band 2-D scans could not determine the exact boundary of the target object, as shown in Figures 9 and 10. It appears likely that significant sidelobes present in both the transmit and the receive antenna patterns resulted in scattered signals appearing when the scanning antenna was off to the side of the landmine and not just when it was overhead. Although the swept-FM 2D-scan imaging systems could only roughly show the target size, as the radiation source frequency increased, more features of the target object could be depicted by the swept-FM system. Compared with the $\mathrm{W}$-band radiation 2D-scan image shown in Figure 9, the F-band 2-D scan imaging could even show the two slots on the objects, significantly benefiting the identification of the target in real situations. 


\section{Below-Ground 2D Imaging Scans}

In the previous sections, under an ideal experimental situation, the sub-Terahertz imaging system demonstrated the ability to accurately position the target and roughly depict the object features. Underground imaging experiments had to be performed to validate the underground imaging of a sub-Terahertz swept-FM system. Since our current F-band source can barely produce sufficient power to penetrate the moist soil, only W-band underground imaging experiments are presented.

Since the microwaves were directed towards a material, energy was reflected or transmitted through the surface or absorbed by it. It is more complicated to image an underground target. In order to mockup an underground imaging situation, mud from a backyard was used as the background material of underground imaging. At the same time, as shown in Figure 11, rock, wooden chips, and sand were added to the mud to mockup the complex situation in the real world. To contain the soil, a $21 \times 21 \times 20$ inch enclosure box was built. It was necessary to mount the enclosure boxes on wheels to facilitate their movement. So that when we removed targets or changed the soil thickness over the targets, the enclosure boxes can be moved far away from the Tx/Rx horns, thus avoiding any contamination of the imaging system. In the following underground imaging experiment, to determine the underground target, a larger region of $30 \mathrm{~cm} \times 30 \mathrm{~cm}$ was scanned. At the same time, a rougher scanning step $(10 \mathrm{~mm} / \mathrm{step})$ and a $500 \mathrm{~ms} / \mathrm{step}$ were used to ensure a reasonable scanning time cost.

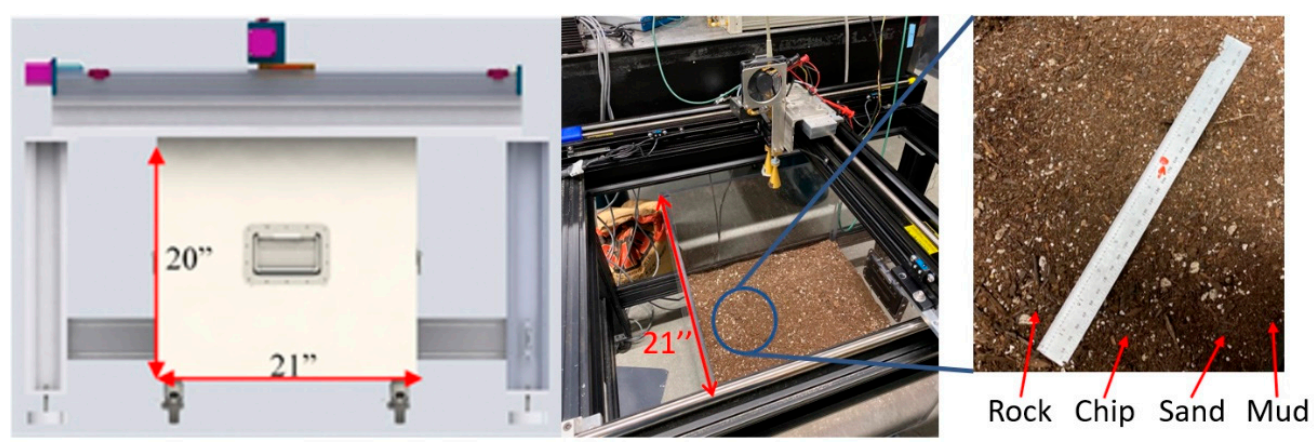

Figure 11. Underground imaging background material and its enclosure box.

We introduced 5 inch $(12.7 \mathrm{~cm})$-thick mixed soil into the box as the imaging background, so that the Rx/Tx antenna horns were $\sim 52 \mathrm{~cm}$ away from the soil surface. The background imaging experimental results, shown in Figure 12a, indicated that the echo signal of the soil surface was found around $7.2 \mathrm{kHz}$. The 2D scanning plot shows a nonuniform echo signal distribution caused by the EM wave reflection from the rock. Then, a target plate was placed on the soil surface, and a 2D-scan was performed. As shown in Figure 12b, a significantly stronger echo signal could be clearly observed near $7.1 \mathrm{kHz}$, indicating the target plate, but a fake imaging signal could also be noticed under the real target, which might be caused by the reflected signal from the enclosure box wall. The $2 \mathrm{D}$-scaning plot could roughly present the shape of the target, and the sidelobes of the $\mathrm{Tx} / \mathrm{Rx}$ antenna patterns resulted in scattered signals affecting imaging quality.

After the on-soil test, $1.5 \mathrm{~cm}$-thick mixed soil was put on the target plate to perform an underground imaging experiment. As shown in Figure 13a, the echo signal of the soil surface and of the target plate were found near $7.0 \mathrm{kHz}$ and $7.1 \mathrm{kHz}$, with different signal amplitude. However, compared with the on-soil experiment, the maximum signal amplitude at the detector dropped from $0.2041 \mathrm{~V}$ to $0.0013 \mathrm{~V}$. The fake imaging was also suppressed due to the absorption by the soil; the reflected signal by the enclosure box wall was subtle. Although the 2D-scanning plot showed the target plate under the soil, the profile of the target could be barely seen. As the thickness of the soil on the target plate increased to $3 \mathrm{~cm}$, the echo signal of the target plate could not be detected; only the surface of the soil echo signal can be seen in Figure 13b. That is because water in the mud and rock 
mixed into the soil significantly absorbed and scattered the sub-Terahertz signal energy. The echo signal of the target was too weak to be distinguished from surface reflection and noise. Consequently, a higher power source is required to let the swept-FM signal penetrate the soil layer covering the target.

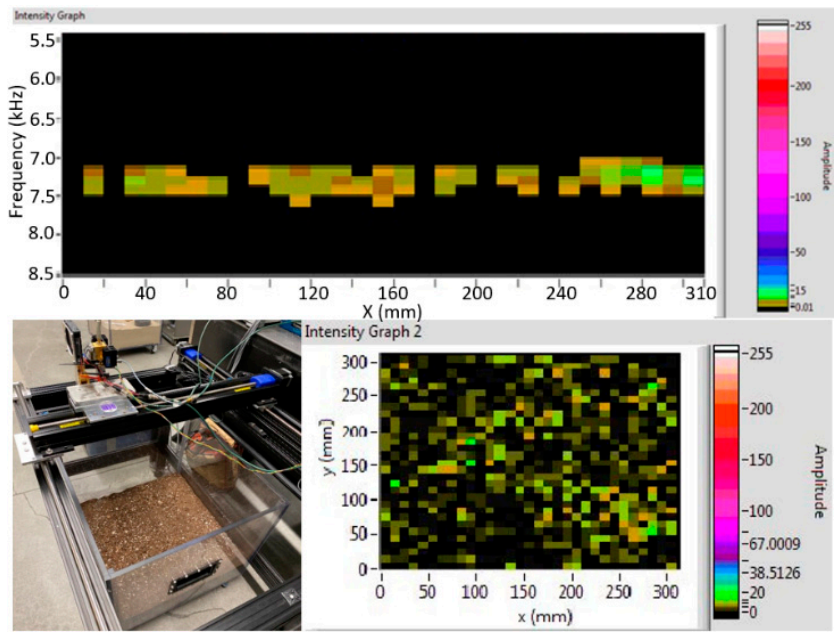

(a)

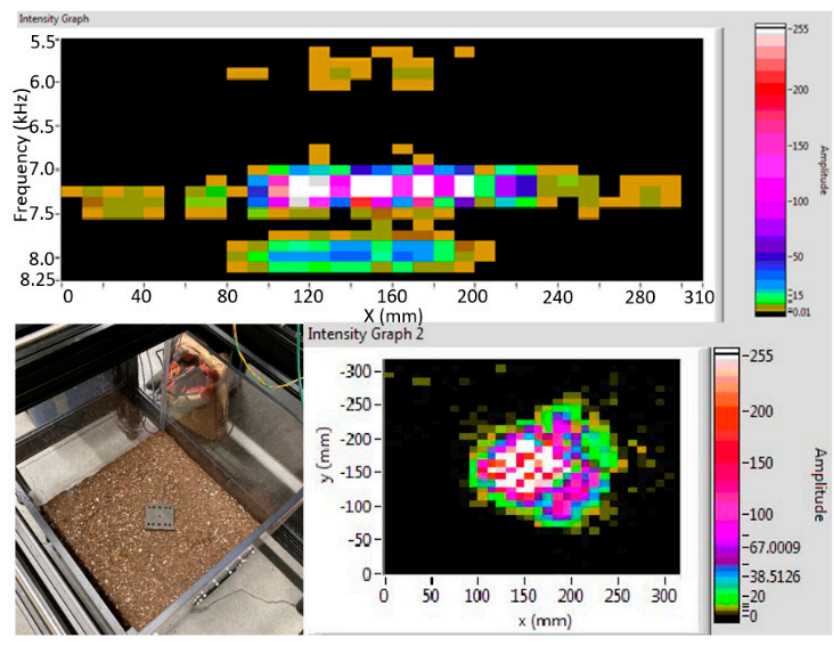

(b)

Figure 12. W-band underground imaging experiment results. (a) Background, (b) target object on soil.

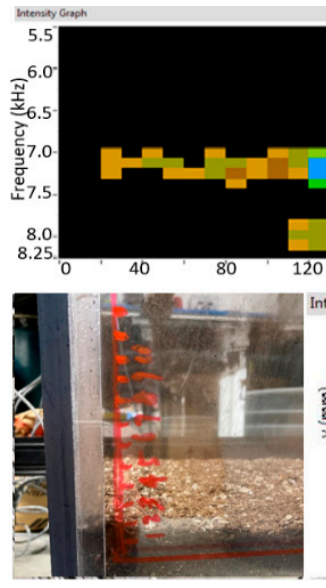

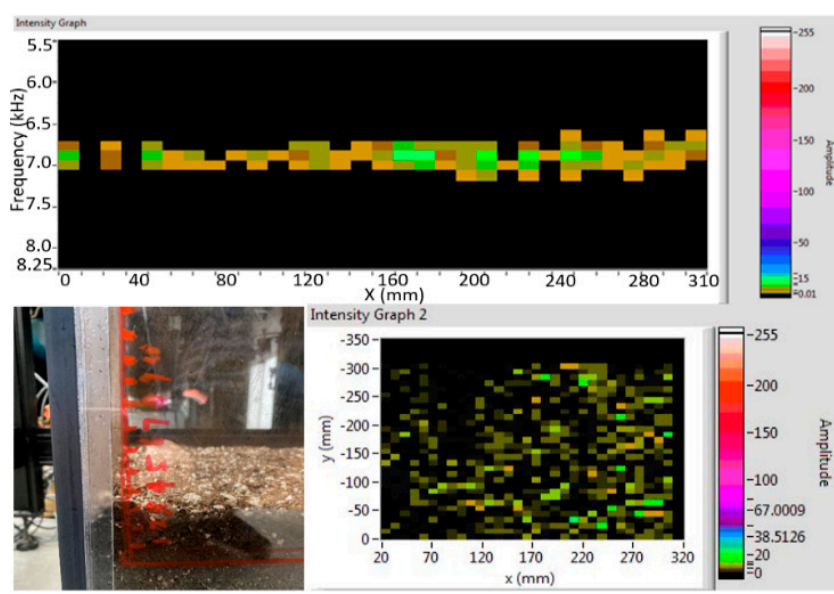

(b)

Figure 13. W-band underground imaging experimental results (a) under $1.5 \mathrm{~cm}$-thick soil, (b) under $3 \mathrm{~cm}$-thick soil.

\section{Discussion}

The in-sand experimental results demonstrated that radiation at millimeter wave frequencies can generate data suitable for underground imaging of metal objects at depths of 9 inches at W-band with $15 \mathrm{~mW}$ of power and of few millimeters at F-band with $1.7 \mathrm{~mW}$ of power. However, the measurements reported in this paper showed only less than $3 \mathrm{~cm}$ penetration depth at $\mathrm{W}$-band in mixed soil with $15 \mathrm{~mW}$ of power. Compared with sand, mixed soil is more similar to soil present in a real situation around a battlefield, but with a stronger attenuation, leading to a shallower detection depth. To prove this assumption, a penetration experiment was performed employing the W-band system.

The attenuation testing system was configured as shown in Figure 14a. The sample box, used to hold test samples of different thicknesses, was placed between the Tx and the Rx horn. The carbon-loaded absorbing material was attached around the Rx horn, inhibiting the interference signal from the metal platform. The testing results, as shown in 
Figure 14b, demonstrated that the attenuation values of sand samples of varying thickness did not change much, and most of the attenuation was caused by the reflection of the surface in this homogeneous medium, since mixed soil is a typical inhomogeneous medium leading to significant scattering, and some of its materials (such as wooden chips and others) may contain water absorbing electromagnetic wave. In contrast to what observed for sand, the attenuation value in mixed soil increased significantly as the thickness of the tested sample increased.

(a)

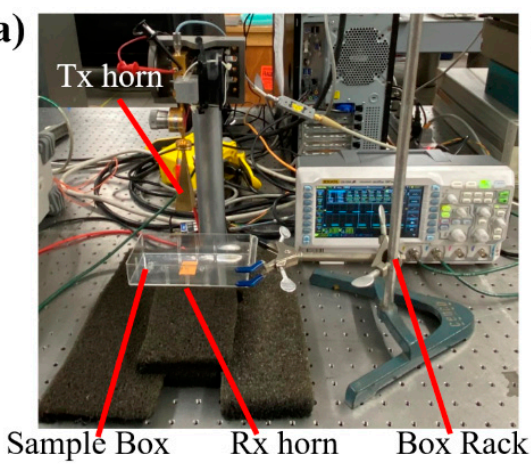

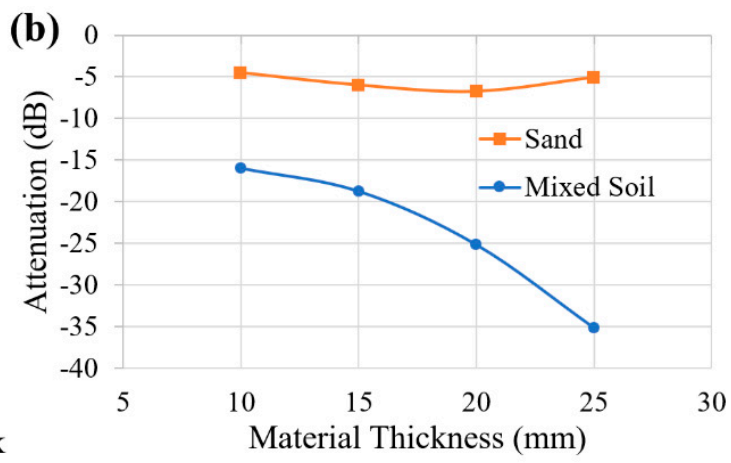

Figure 14. W-band attenuation (a) testing platform and (b) related experiment results.

The previous experiments showed that imaging at 100-150 GHz frequency is feasible for underground applications; however, limited availability of power and the sensitivity of the receive components significantly restrict the penetration capability of this approach. However, with recent significant advancements in medium- to high-power ultra-compact sources, this problem can be readily addressed. For example, we have recently developed a $1 \mathrm{~kW}$ low-voltage ultra-compact W-band klystron (LUWK), as shown in Figure 15a, weighing fewer than 10 pounds, making it feasible for a readily transportable RF source [4]. A similar fabrication technology and focusing system could be applied to develop a TWT with significantly broader bandwidth as the high-power source extending the penetration depth. In parallel, we also demonstrated that the SoC technology [5,11], shown in Figure $15 b$, originally developed for plasma diagnostics, is also well suited for improving the sensitivity to the echo of an underground imaging signal. We are trying to integrate those high-power devices and sensitive components into a landmine imaging system allowing for a sufficiently deep penetration into the soil.

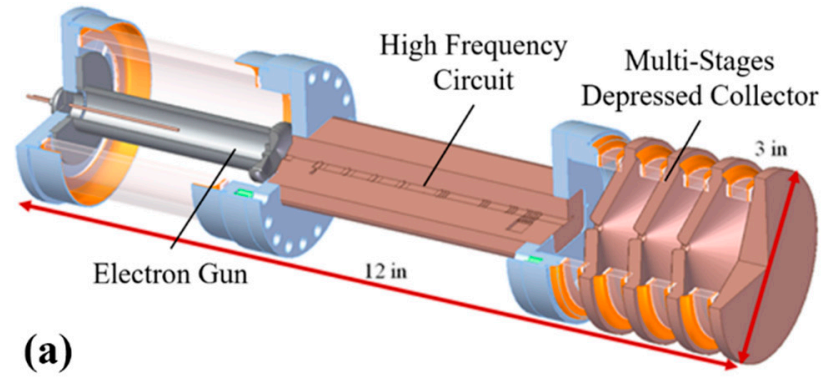

(b)

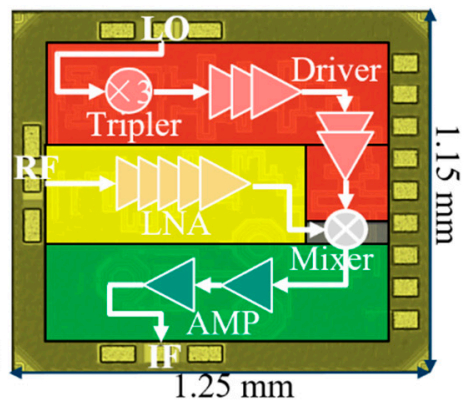

Figure 15. General view of the (a) W-band LUWK and (b) F-band SoC Chip.

Author Contributions: Y.Z., C.D. and M.G. were responsible for experimental demonstrations, systems design, implementation, formal results analysis, and composition of the paper; D.G. and N.C.L.J. were responsible for program conceptualization, project execution, supervision, administration, and funding acquisition. All authors have read and agreed to the published version of the manuscript.

Funding: This research was supported by the U.S. Army's Night Vision Directorate (NVESD) (contract number 18-0035-IA) and Army Research Lab (ARL) W-band Source Demonstrator (contract number 17-054). 
Data Availability Statement: The data presented in this study are available in this article.

Conflicts of Interest: The authors declare no conflict of interest.

\section{References}

1. Appleby, R.; Anderton, R. Millimeter-wave and submillimeterwave imaging for security and surveillance. Proc. IEEE 2007, 95, 1683-1690. [CrossRef]

2. Gamzina, D.; Barnett, L.R.; Ravani, B.; Luhmann, N.C., Jr. Mechanical Design and Manufacturing of W-Band Sheet Beam Klystron. IEEE Trans. Electron. Devices 2017, 64, 2675-2682. [CrossRef]

3. Shin, Y.; Barnett, L.R.; Luhmann, N.C., Jr. Particle-in-Cell (PIC) Simulation Analysis of a Quasi-Optical W-Band Sheet Beam Klystron. IEEE Trans. Electron. Devices 2011, 58, 251-258. [CrossRef]

4. Zheng, Y.; Sy, A.; Weatherford, B.; Luhmann, N.C.; Gamzina, D. Multioutput Circuit for Low Voltage Ultracompact W-Band Klystron. IEEE Trans. Electron. Devices 2020, 67, 3821-3827. [CrossRef]

5. Yu, J.-H.; Chang, Y.-T.; Lin, K.-Y.; Chang, C.-C.; Chang, S.-F.; Ye, Y.; Pham, A.V.; Tobias, B.J.; Zhu, Y.; Domier, C.W.; et al. Millimeter-wave system-on-chip advancement for fusion plasma diagnostics. Rev. Sci. Instrum. 2018, 89, 10H108. [CrossRef] [PubMed]

6. Daniels, D.J. Ground Penetrating Radar. In Encyclopedia of RF and Microwave Engineering; Chang, K., Ed.; John Wiley \& Sons, Inc.: Hoboken, NJ, USA, 2005.

7. Martinez-Lorenzo, J.; Rappaport, C.; Quvira, F. Physical limitations on detecting tunnels using underground-focusing spotlight synthetic aperture radar. IEEE Trans. Geosci. Remote Sens. 2011, 49, 65-70. [CrossRef]

8. Fernández, M.; López, Y.; Valdés, B.; Vaqueiro, Y.; Adrés, F.; García, A. Synthetic Aperture Radar Imaging System for Landmine Detection Using a Ground Penetrating Radar on Board a Unmanned Aerial Vehicle. IEEE Access 2018, 6, 45100-45112. [CrossRef]

9. Garcia-Fernandez, M.; Alvarez-Lopez, Y.; Las Heras, F. Autonomous Airborne 3D SAR Imaging System for Subsurface Sensing: UWB-GPR on Board a UAV for Landmine and IED Detection. Remote Sens. 2019, 11, 2357. [CrossRef]

10. Topcon B111 Receiver. Available online: https://www.topconpositioning.com/oem-componentstechnology/gnss-components/ b111 (accessed on 13 February 2021).

11. Zhu, Y.L.; Yu, J.H.; Yu, G.; Chen, Y.; Tobias, B.; Diallo, A.; Kramer, G.; Ren, Y.; Tang, W.; Dong, G.; et al. System-on-chip upgrade of millimeter-wave imaging diagnostics for fusion plasma. Rev. Sci. Inst. 2021, 92, 053522. [CrossRef] [PubMed] 